\title{
THE NORMAL COMPLETIONS OF CERTAIN PARTIALLY ORDERED VECTOR SPACES
}

\author{
ALAN G. WATERMAN ${ }^{1}$
}

ABstract. It is shown that the normal completions of certain partially ordered vector spaces are the same as certain other normal completions determined by Dilworth.

Among the standard finite-dimensional partially ordered real vector spaces are the space $A_{n}=R^{n+1}$, with the positive cone being the set of vectors $\left(x_{0}, x_{1}, \cdots, x_{n}\right)$ such that $x_{0} \geqq 0$ and $x_{0}^{2} \geqq x_{1}^{2}+\cdots$ $+x_{n}^{2}$, and the space $B_{n}$ of symmetric $n \times n$ matrices, with the positive cone consisting of the nonnegative definite matrices. Also of interest is $F_{n}$, the free vector lattice on $n$ generators, which has recently been determined by Kirby Baker [1].

Since these spaces are completely integrally closed, their normal completions (also called the completion by cuts) exist and are complete vector lattices (Fuchs [2, p. 95, Theorem 19]). The object of this note is to show that these spaces have the same normal completion as the spaces $C(T)$, for certain topological spaces $T$, which Dilworth [3] showed have isomorphic completions.

Let $X$ and $Y$ be the vectors $\left(x_{1}, \cdots, x_{n}\right)$ and $\left(y_{1}, \cdots, y_{n}\right)$ in $R^{n}$, $X \cdot Y=x_{1} y_{1}+\cdots+x_{n} y_{n}$, and $|X|=(X \cdot X)^{1 / 2}$. Then $A_{n}$ consists of the pairs $\left(x_{0}, X\right)$, and $\left(x_{0}, X\right) \geqq 0 \leftrightarrow x_{0} \geqq|X|$.

Let $S^{n-1}=\left\{Y \in R^{n}:|Y|=1\right\}$ be the unit sphere in $R^{n}$. Define $\phi: A^{n} \rightarrow C\left(S^{n-1}\right)$ by $\phi\left(x_{0}, X\right)(Y)=x_{0}+X \cdot Y$.

Lemma 1. $\phi$ is an embedding of $A_{n}$ into $C\left(S^{n-1}\right)$.

Proof. $\phi$ is obviously linear. $\phi\left(x_{0}, X\right) \geqq 0$ iff $\forall Y \in S^{n-1}, \phi\left(x_{0}, X\right)(Y)$ $=x_{0}+X \cdot Y \geqq 0$ iff $\forall Y \in S^{n-1}, x_{0} \geqq-X \cdot Y$. Since the maximum of $-X \cdot Y$ over $Y \in S^{n-1}$ is $|X|$, attained when $Y$ is opposite in direction to $X$, it follows that $\phi\left(x_{0}, X\right) \geqq 0$ iff $x_{0} \geqq|X|$ iff $\left(x_{0}, X\right) \geqq 0$.

Leмma 2. The normal completion of $A_{n}$ contains $C\left(S^{n-1}\right)$.

Proof. Let $f \in C\left(S^{n-1}\right), \quad Y_{0} \in S^{n-1}$, and $u<f\left(Y_{0}\right)$. Let $m$ be the

Received by the editors March 21, 1967 and, in revised form, March 22, 1969.

A MS Subject Classifications. Primary 0680.

Key Words and Phrases. Normal completion (completion by cuts), partially ordered vector spaces.

1 This research was supported by Grant GP 2184 from the National Science Foundation. 
smaller of $u$ and the minimum value of $f$. By continuity of $f, \exists \delta>0$ $\left|Y-Y_{0}\right|<\delta \rightarrow f(Y)>u$. We notice that, by the Euclidean geometry of the circle, $\left|Y-Y_{0}\right|<\delta \leftrightarrow Y_{0} \cdot Y>1-\delta^{2} / 2$. Let $r=2(u-m) / \delta^{2}$, $x_{0}=u-r, X=r Y_{0}$. Then, if $\left|Y-Y_{0}\right|<\delta$, then $\phi\left(x_{0}, X\right)(Y)=x_{0}$ $+X \cdot Y=u-r+r Y_{0} \cdot Y \leqq u-r+r=u<f(Y)$, whereas if $\left|Y-Y_{0}\right| \geqq \delta$, then $Y_{0} \cdot Y \leqq 1-\delta^{2} / 2$ and $\phi\left(x_{0}, \quad X\right)(Y)=u-r+r Y_{0} \cdot Y \leqq u-r$ $+r\left(1-\delta^{2} / 2\right)=m \leqq f(Y)$. Thus $\phi\left(x_{0}, X\right) \leqq f$. Also $\phi\left(x_{0}, X\right)\left(Y_{0}\right)=u-r$ $+r Y_{0} \cdot Y_{0}=u$. This is true for all real $u<f\left(Y_{0}\right)$, hence $f\left(Y_{0}\right)=$ lub $\left\{\phi\left(x_{0}, \quad X\right)\left(Y_{0}\right): \phi\left(x_{0}, X\right) \leqq f\right\}$. This again is true for all $Y_{0} \in S^{n-1}$, hence $f=\operatorname{lub}\left\{\phi\left(x_{0}, X\right): \phi\left(x_{0}, X\right) \leqq f\right\}$. Similarly $f=$ $\operatorname{glb}\left\{\phi\left(x_{0}, X\right): \phi\left(x_{0}, X\right) \geqq f\right\}$. Thus $f$ is in the normal completion of $A_{n}$.

THEOREM IA. $\bar{A}_{n}$, the normal completion of $A_{n}$, is the same as $\bar{C}\left(S^{n-1}\right)$, the normal completion of $C\left(S^{n-1}\right)$.

Proof. By Lemmas 1 and $2, A_{n} \subseteq C\left(S^{n-1}\right) \subseteq \bar{A}_{n}$.

Let us turn now to the space $B_{n}$. The elements of $B_{n}$ may be considered as selfadjoint linear operators $U$ on $R^{n}$. Define $\psi: B_{n} \rightarrow C\left(S^{n-1}\right)$ by $\psi(U)(Y)=U(Y) \cdot Y$. Since $\psi(U)(Y)=\psi(U)(-Y), \psi$ may be considered as a function from $B_{n}$ to $C\left(P^{n-1}\right)$, where $P^{n-1}$ is the $(n-1)$ dimensional projective space.

Lemma 3. $\psi$ is an embedding of $B_{n}$ into $C\left(P^{n-1}\right)$.

Proof. $\psi$ is obviously linear. $\psi(U) \geqq 0$ iff $\forall Y \in P^{n-1}, \psi(U)(Y) \geqq 0$ iff $\forall Y \in S^{n-1}, \psi(U)(Y) \geqq 0$ iff $\forall Y \in R^{n}, U(Y) \cdot Y \geqq 0$ iff $U$ is nonnegative definite iff $U \geqq 0$ in $B_{n}$.

Lemma 4. The normal completion of $B_{n}$ contains $C\left(P^{n-1}\right)$.

Proof. Let $f \in C\left(P^{n-1}\right)$ and let $f$ be considered thereby as an element of $C\left(S^{n-1}\right)$, let $Y_{0} \in S^{n-1}$, and $u<f\left(Y_{0}\right)$. Let $m$ be the smaller of $u$ and the minimum value of $f$. By continuity of $f$, as before, $\exists \delta>0$ $\left|Y-Y_{0}\right|<\delta \rightarrow f(Y)>u$. Let $s=(u-m) /\left(\delta^{2}-\delta^{4} / 4\right)$, and for $X \in R^{n}$, let $U(X)=(u-s) X+s\left(Y_{0} \cdot X\right) Y_{0}$. Then, if $\left|Y-Y_{0}\right|<\delta$, then $\psi(U)(Y)=U(Y) \cdot Y=(u-s) Y \cdot Y+s\left(Y_{0} \cdot Y\right)^{2} \leqq u-s+s=u<f(Y)$, and similarly if $\left|Y-\left(-Y_{0}\right)\right|<\delta$, whereas if $\left|Y \pm Y_{0}\right| \geqq \delta$, then $\psi(U)(Y)$ $=(u-s) Y \cdot Y+s\left(Y_{0} \cdot Y\right)^{2} \leqq u-s+s\left(1-\delta^{2} / 2\right)^{2}=m \leqq f(Y)$. Also $\psi(U)\left(Y_{0}\right)=u$. As before, since this is true for all $Y_{0}$ and $u, f$ is in the normal completion of $B_{n}$.

THEOREM IB. $\bar{B}_{n}$, the normal completion of $B_{n}$, is the same as $\bar{C}\left(P^{n-1}\right)$, the normal completion of $C\left(P^{n-1}\right)$. 
Proof. By Lemmas 3 and $4, B_{n} \subseteq C\left(P^{n-1}\right) \subseteq \bar{B}_{n}$.

Finally, consider $F_{n}$, the free vector lattice on $n$ generators. This has recently been determined as the vector lattice of continuous functions on $R^{n}$ generated by the coordinate projections $g_{i}: g_{i}(Y)=y_{i}$. These functions are those which are positively homogeneous of degree one and piecewise linear on polyhedral cones with vertex at the origin. Since such functions are determined by their restrictions to $S^{n-1}, F_{n}$ may be embedded in $C\left(S^{n-1}\right)$.

\section{Lemma 5. The normal completion of $F_{n}$ contains $C\left(S^{n-1}\right)$ for $n \geqq 2$.}

Proof. Let $f \in C\left(S^{n-1}\right), Y_{0}=(1,0, \cdots, 0)$, and $u<f\left(Y_{0}\right)$. Let $m$ be the smaller of $u$ and the minimum value of $f$. Again by continuity of $f$, $\exists \delta>0\left|Y-Y_{0}\right|<\delta \rightarrow f(Y)>u$. Let $\quad p=\left(\left(\delta^{2}-\delta^{4} / 4\right) /(n-1)\right)^{1 / 2}$, $t=(u-m) / p, q=\max \left(0, u,-m /\left(1-\delta^{2} / 2\right)\right)$. Let $h_{i}=u g_{1}-t g_{i}, \quad k_{i}$ $=u g_{1}+t g_{i}, \quad H=h_{2} \wedge \cdots \wedge h_{n} \wedge k_{2} \wedge \cdots \wedge k_{n} \wedge q g_{1} \wedge u g_{1}$. If $\left|y_{i}\right|<p$ for $2 \leqq i \leqq n$, then $\left|y_{1}\right|>1-\delta^{2} / 2$. Thus, if $y_{i}>p$, then $H(Y) \leqq h_{i}(Y)$ $=u y_{1}-t y_{i} \leqq u-(u-m)=m \leqq f(Y)$, and similarly if $y_{i}<-p$, and if $y_{1}<-1+\delta^{2} / 2$, then $H(Y) \leqq q g_{1}(Y)=q y_{1} \leqq m \leqq f(Y)$, whereas if $y_{1}>1-\delta^{2} / 2$, then $\left|Y-Y_{0}\right|<\delta$, and $H(Y) \leqq u g_{1}(Y)=u y_{1} \leqq u<f(Y)$. Also $H\left(Y_{0}\right)=u$. This is true for all real $u<f\left(Y_{0}\right)$, hence $f\left(Y_{0}\right)=$ lub $\left\{H\left(Y_{0}\right): H \in F_{n}, H \leqq f\right\}$. But $F_{n}$ and $C\left(S^{n-1}\right)$ are invariant with respect to rotations of $S^{n-1}$, hence for all $Y \in S^{n-1}, f(Y)=$ lub $\left\{H(Y): H \in F_{n}, H \leqq f\right\}$, hence $f=\operatorname{lub}\left\{H: H \in F_{n}, H \leqq f\right\}$. Similarly, $f=\operatorname{glb}\left\{H: H \in F_{n}, H \geqq f\right\}$. Thus $f$ is in the normal completion of $F_{n}$.

THEOREM IF. $\bar{F}_{n}$, the normal completion of $F_{n}$, is the same as $\bar{C}\left(S^{n-1}\right)$.

Proof. By Lemma 5, $F_{n} \subseteq C\left(S^{n-1}\right) \subseteq \bar{F}_{n}$.

The normal completions of $A_{n}, B_{n}$, and $F_{n}$ have thus been determined as the completions of $C(T)$ for certain compact Hausdorff spaces $T$. It has been shown by Dilworth [3, Theorem 7.1] that the spaces $\bar{C}(T)$ are the same for all nonempty completely regular secondcountable spaces without isolated points, and that $\bar{C}(T)$ may be described as the lattice of normal upper semicontinuous functions on $T$. Dilworth also describes this lattice as the lattice of continuous functions on a certain Stone space. An alternative characterization has been given by Semadeni [4] and Ramsay [5] as the lattice of bounded functions $h$ on $T$ such that the restriction of $h$ to some residual set is continuous, modulo those which vanish on some residual set.

Thus we have: 
Theorem I. The normal completions of $A_{n}, B_{n}$, and $F_{n}$ are isomorphic to the spaces $\bar{C}(T)$ described above, for $n \geqq 2$. $A_{1}, B_{1}$, and $F_{1}$ are complete.

\section{REFERENCES}

1. Kirby Baker, Free vector lattices, Canad. J. Math. 20 (1968), 58-66. MR 37 $\# 123$.

2. L. Fuchs, Partially ordered algebraic systems, Pergamon Press, New York and Addison-Wesley, Reading, Mass., 1963. MR 30 \#2090.

3. R. P. Dilworth, The normal completion of the lattice of continuous functions, Trans. Amer. Math. Soc. 68 (1950), 427-438. MR 11, 647.

4. Z. Semadeni, Spaces of continuous functions on compact sets, Advances in Math. 1 (1965), no. 3, 319-382. MR 32 \#2937.

5. Arlan Ramsay, Personal communication (unpublished).

Harvard University, Cambridge, Massachusetts 02138 